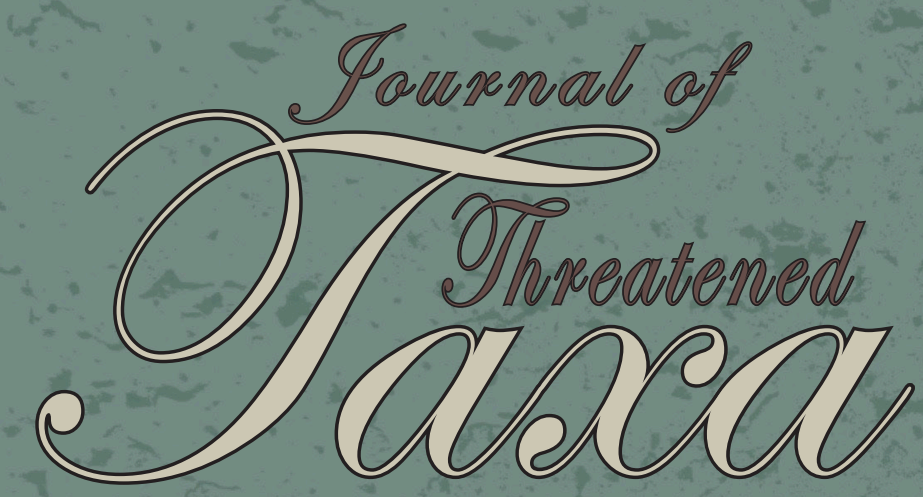

Building evidence for conservation globally
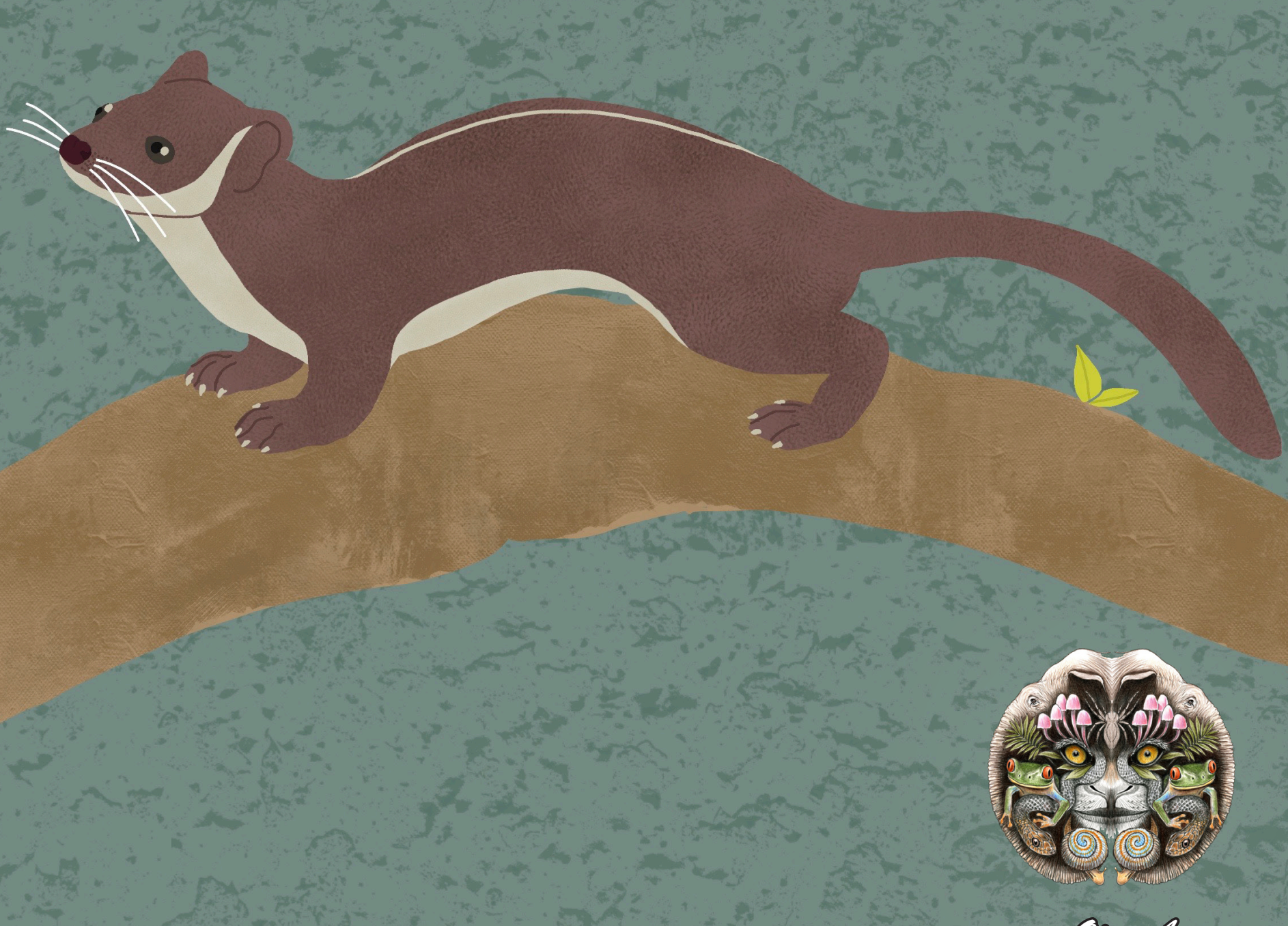

Qpecosecess

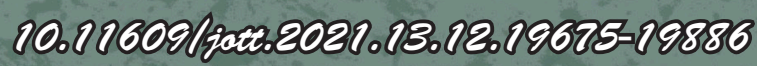
cocosurthreatecredtassararg

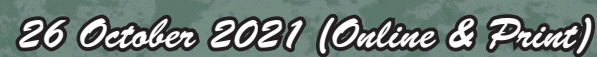

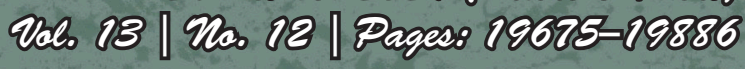




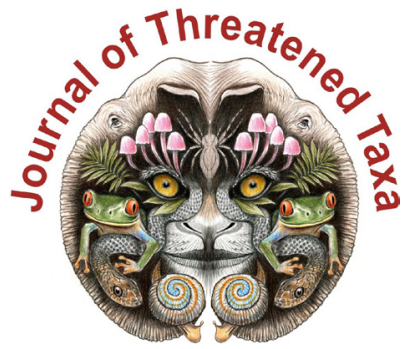

ISSN 0974-7907 (Online); ISSN $0974-7893$ (Print)

Publisher

Host

Wildlife Information Liaison Development Society

www.wild.zooreach.org

Zoo Outreach Organization www.zooreach.org

No. 12, Thiruvannamalai Nagar, Saravanampatti - Kalapatti Road, Saravanampatti, Coimbatore, Tamil Nadu 641035, India

Ph: +91 9385339863 | www.threatenedtaxa.org

Email: sanjay@threatenedtaxa.org

EDITORS

\section{Founder \& Chief Editor}

Dr. Sanjay Molur

Wildlife Information Liaison Development (WILD) Society \& Zoo Outreach Organization (ZOO)

12 Thiruvannamalai Nagar, Saravanampatti, Coimbatore, Tamil Nadu 641035, India

\section{Deputy Chief Edito}

Dr. Neelesh Dahanukar

Noida, Uttar Pradesh, India

\section{Managing Editor}

Mr. B. Ravichandran, WILD/ZOO, Coimbatore, India

\section{Associate Editors}

Dr. Mandar Paingankar, Government Science College Gadchiroli, Maharashtra 442605, India Dr. Ulrike Streicher, Wildlife Veterinarian, Eugene, Oregon, USA

Ms. Priyanka Iyer, ZOO/WILD, Coimbatore, Tamil Nadu 641035, India

Dr. B. A. Daniel, $200 /$ WIID, Coimbatore, Tamil Nadu 641035, India

\section{Editorial Board}

Dr. Russel Mittermeie

Executive Vice Chair, Conservation International, Arlington, Virginia 22202, USA

\section{Prof. Mewa Singh Ph.D., FASc, FNA, FNASc, FNAPsy}

Ramanna Fellow and Life-Long Distinguished Professor, Biopsychology Laboratory, and Institute of Excellence, University of Mysore, Mysuru, Karnataka 570006, India; Honorary Professor, Jawaharlal Nehru Centre for Advanced Scientific Research, Bangalore; and Adjunct Professor, National Institute of Advanced Studies, Bangalore

\section{Stephen D. Nash}

Scientific Illustrator, Conservation International, Dept. of Anatomical Sciences, Health Sciences Center, T-8, Room 045, Stony Brook University, Stony Brook, NY 11794-8081, USA

\section{Dr. Fred Pluthero}

Toronto, Canada

\section{Dr. Priya Davidar}

Sigur Nature Trust, Chadapatti, Mavinhalla PO, Nilgiris, Tamil Nadu 643223, India

\section{Dr. Martin Fisher}

Senior Associate Professor, Battcock Centre for Experimental Astrophysics, Cavendish

Laboratory, JJ Thomson Avenue, Cambridge CB3 OHE, UK

\section{Dr. John Fellowes}

Honorary Assistant Professor, The Kadoorie Institute, 8/F, T.T. Tsui Building, The University of Hong Kong, Pokfulam Road, Hong Kong

\section{Prof. Dr. Mirco Solé}

Universidade Estadual de Santa Cruz, Departamento de Ciências Biológicas, Vice-coordenado do Programa de Pós-Graduação em Zoologia, Rodovia Ilhéus/Itabuna, Km 16 (45662-000)

Salobrinho, Ilhéus - Bahia - Brasil

\section{Dr. Rajeev Raghavan}

Professor of Taxonomy, Kerala University of Fisheries \& Ocean Studies, Kochi, Kerala, India

\section{English Editors}

Mrs. Mira Bhojwani, Pune, India

Dr. Fred Pluthero, Toronto, Canad

Mr. P. Ilangovan, Chennai, India

Web Maintenance

Mrs. Latha G. Ravikumar, ZOO/WILD, Coimbatore, India

\section{Typesetting}

Mr. Arul Jagadish, ZOO, Coimbatore, India

Mrs. Radhika, ZOO, Coimbatore, India

Mrs. Geetha, ZOO, Coimbatore India

\section{Fundraising/Communications}

Mrs. Payal B. Molur, Coimbatore, India

Subject Editors 2018-2020

Fungi

Dr. B. Shivaraju, Bengaluru, Karnataka, India

Dr. R.K. Verma, Tropical Forest Research Institute, Jabalpur, India

Dr. Vatsavaya S. Raju, Kakatiay University, Warangal, Andhra Pradesh, India

Dr. M. Krishnappa, Jnana Sahyadri, Kuvempu University, Shimoga, Karnataka, India

Dr. K.R. Sridhar, Mangalore University, Mangalagangotri, Mangalore, Karnataka, India

Dr. Gunjan Biswas, Vidyasagar University, Midnapore, West Bengal, India

Plants

Dr. G.P. Sinha, Botanical Survey of India, Allahabad, India

Dr. N.P. Balakrishnan, Ret. Joint Director, BSI, Coimbatore, India

Dr. Shonil Bhagwat, Open University and University of Oxford, UK

Prof. D.J. Bhat, Retd. Professor, Goa University, Goa, India

Dr. Ferdinando Boero, Università del Salento, Lecce, Italy

Dr. Dale R. Calder, Royal Ontaro Museum, Toronto, Ontario, Canada

Dr. Cleofas Cervancia, Univ. of Philippines Los Baños College Laguna, Philippines

Dr. F.B. Vincent Florens, University of Mauritius, Mauritius

Dr. Merlin Franco, Curtin University, Malaysia

Dr. V. Irudayaraj, St. Xavier's College, Palayamkottai, Tamil Nadu, India

Dr. B.S. Kholia, Botanical Survey of India, Gangtok, Sikkim, India

Dr. Pankaj Kumar, Kadoorie Farm and Botanic Garden Corporation, Hong Kong S.A.R., China

Dr. V. Sampath Kumar, Botanical Survey of India, Howrah, West Bengal, India

Dr. A.J. Solomon Raju, Andhra University, Visakhapatnam, India

Dr. Vijayasankar Raman, University of Mississippi, USA

Dr. B. Ravi Prasad Rao, Sri Krishnadevaraya University, Anantpur, India

Dr. K. Ravikumar, FRLHT, Bengaluru, Karnataka, India

Dr. Aparna Watve, Pune, Maharashtra, India

Dr. Qiang Liu, Xishuangbanna Tropical Botanical Garden, Yunnan, China

Dr. Noor Azhar Mohamed Shazili, Universiti Malaysia Terengganu, Kuala Terengganu, Malaysia Dr. M.K. Vasudeva Rao, Shiv Ranjani Housing Society, Pune, Maharashtra, India

Prof. A.J. Solomon Raju, Andhra University, Visakhapatnam, India

Dr. Mandar Datar, Agharkar Research Institute, Pune, Maharashtra, India

Dr. M.K. Janarthanam, Goa University, Goa, India

Dr. K. Karthigeyan, Botanical Survey of India, India

Dr. Errol Vela, University of Montpellier, Montpellier, France

Dr. P. Lakshminarasimhan, Botanical Survey of India, Howrah, India

Dr. Larry R. Noblick, Montgomery Botanical Center, Miami, USA

Dr. K. Haridasan, Pallavur, Palakkad District, Kerala, India

Dr. Analinda Manila-Fajard, University of the Philippines Los Banos, Laguna, Philippines

Dr. P.A. Sinu, Central University of Kerala, Kasaragod, Kerala, India

Dr. Afroz Alam, Banasthali Vidyapith (accredited A grade by NAAC), Rajasthan, India

Dr. K.P. Rajesh, Zamorin's Guruvayurappan College, GA College PO, Kozhikode, Kerala, India

Dr. David E. Boufford, Harvard University Herbaria, Cambridge, MA 02138-2020, USA

Dr. Ritesh Kumar Choudhary, Agharkar Research Institute, Pune, Maharashtra, India

Dr. Navendu Page, Wildlife Institute of India, Chandrabani, Dehradun, Uttarakhand, India

Invertebrates

Dr. R.K. Avasthi, Rohtak University, Haryana, India

Dr. D.B. Bastawade, Maharashtra, India

Dr. Partha Pratim Bhattacharjee, Tripura University, Suryamaninagar, India

Dr. Kailash Chandra, Zoological Survey of India, Jabalpur, Madhya Pradesh, India

Dr. Ansie Dippenaar-Schoeman, University of Pretoria, Queenswood, South Africa

Dr. Rory Dow, National Museum of natural History Naturalis, The Netherlands

Dr. Brian Fisher, California Academy of Sciences, USA

Dr. Richard Gallon, llandudno, North Wales, LL30 1UP

Dr. Hemant V. Ghate, Modern College, Pune, India

Dr. M. Monwar Hossain, Jahangirnagar University, Dhaka, Bangladesh

Mr. Jatishwor Singh Irungbam, Biology Centre CAS, Branišovská, Czech Republic.

Dr. lan J. Kitching, Natural History Museum, Cromwell Road, UK

Dr. George Mathew, Kerala Forest Research Institute, Peechi, India

Dr. John Noyes, Natural History Museum, London, UK

For Focus, Scope, Aims, and Policies, visit https://threatenedtaxa.org/index.php/JoTT/aims_scope
For Article Submission Guidelines, visit https://threatenedtaxa.org/index.php/JoTT/about/submissions
For Policies against Scientific Misconduct, visit https://threatenedtaxa.org/index.php/JoTT/policies_various

continued on the back inside cover 


\title{
Notes on the extended distribution of Impatiens megamalayana, a recently described balsam in Western Ghats, India
}

\author{
Anoop P. Balan ${ }^{1}$ (D) \& A.J. Robi ${ }^{2}$ (1) \\ 1,2 Bishop Abraham Memorial College, Thuruthicad, Pathanamthitta, Kerala 689597, India. \\ ${ }^{1}$ anooppb01@gmail.com (corresponding author), ${ }^{2}$ ajrobin80@gmail.com
}

The balsam family Balsaminaceae includes two genera and more than 1,000 species of fleshy herbs. Hydrocera Blume ex Wight \& Arn. is a monotypic genus with a single species $H$. triflora (L.) Wight \& Arn., and is distributed from Sri Lanka, India to Java, southern Borneo, and Sulawesi (Grey-Wilson 1980). Impatiens L., the other genus of the family is highly diversified and is known to have five distinct diversity hotspots, viz., tropical Africa, Madagascar, Southern India, Sri Lanka, the eastern Himalaya, and southeastern Asia (Yuan et al. 2004). In India, the genus is represented by more than 210 species, mainly distributed in the eastern Himalaya, the northeastern states, and the Western Ghats (Bhaskar 2012). The Western Ghats is one of the major centres of diversification of Impatiens. Nayar et al. (2014) enumerated 106 taxa of Impatiens from the Western Ghats of which 97 are exclusive endemics. More than 30 species have been discovered from various parts of the Western Ghats during the last 10 years (Kumar et al. 2011; Dessai \& Janarthanam 2011; Narayanan et al. 2011, 2012a,b, 2013; Hareesh et al. 2015a; Prabhukumar et al. 2015a,b, 2016, 2017; Ramasubbu et al. 2015a,b, 2017, 2020a,b; Chhabra et al. 2016; Bhaskar \& Sringeswar 2017; Manudev et al. 2017; Mani \& Thomas 2017; Mani et al. 2018, 2020; Arigela et al. 2019; Salish et al. 2019;
Subbiah \& Vellingiri 2019; Vishnu et al. 2020 \& 2021; Arya et al. 2021). Impatiens species are usually seen in open, wet grasslands and rocky hill slopes at higher elevations and are highly sensitive to micro-climatic conditions. Major populations of Impatiens in the Western Ghats are outside protected forest areas and are facing severe threat due to habitat loss and changes in climatic conditions.

Kerala, one of the smallest states in India is rich in its balsam diversity. Sasidharan (2011) enlisted 80 species from Kerala, of which 17 are endemic to the state. Since then, 24 species have been added to the balsam flora of the state including a new distributional record (Anil Kumar et al. 2011; Narayanan et al. 2011, 2012a,b, 2013; Hareesh et al. 2015a,b; Prabhukumar et al. 2015a,b; Ramasubbu 2015b; Prabhukumar et al. 2016, 2017; Manudev et al. 2017; Mani \& Thomas 2017; Mani et al. 2018, 2020; Salish et al. 2019; Vishnu et al. 2020 \& 2021; Arya et al. 2021). Joe et al. 2017 elevated Impatiens rufescens var. agastyamalayensis to species level, i.e., I. agastyamalayensis (Bhaskar) Joe, Bhaskar $\&$ Sabu. Thus, the total number of Impatiens in Kerala state reached 107 . While exploring the Urumbikkara hills of Idukki district in central Kerala, the authors came across a small, delicate Impatiens with pale red

Citation: Balan, A.P. \& A.J. Robi (2021). Notes on the extended distribution of Impatiens megamalayana, a recently described balsam in Western Ghats, India. Journal of Threatened Taxa 13(12): 19878-19883. https://doi.org/10.11609/jott.7034.13.12.19878-19883

Copyright: (C) Balan \& Robi 2021. Creative Commons Attribution 4.0 International License. JoTT allows unrestricted use, reproduction, and distribution of this article in any medium by providing adequate credit to the author(s) and the source of publication.

Funding: None.

Competing interests: The authors declare no competing interests

Acknowledgements: The authors are grateful to the Principal, Bishop Abraham Memorial College, Thuruthicad, Pathanamthitta, Kerala for the facilities and support. 
flowers. Upon critical examination it was identified as I. megamalayana Ramas., a recently described balsam from the Megamalai hills of Tamil Nadu. This is the first report of occurrence of this narrow endemic species outside its type locality. Detailed description, images, distribution map and notes on habitat, ecology and conservation status are provided for easy identification and a better understanding of the species. An updated checklist of the balsams of Kerala state is also furnished for future reference.

\section{Taxonomic Treatment}

Impatiens megamalayana

Ramas., Phytotaxa 302(2): 193. 2018.

(Image 1)

Annual, succulent, erect, herbs, 25-40 cm tall; stem purplish, cylindrical with 3-5 ridges, often branched, glabrous, nodes slightly swollen, internodes $4-6 \mathrm{~cm}$ long. Leaves opposite, decussate; petiole $0.3-0.4 \mathrm{~mm}$ long, glabrous, with 1-2 pairs of extra-petiolar glands; lamina linear-ensiform, $2.6-5.0 \times 0.5-0.8 \mathrm{~cm}$, acuteshortly acuminate at apex, truncate or slightly cordate at base, sparsely hairy above along the midrib, smooth and sometimes purplish below, margins distinctly serrate, midrib distinct, lateral veins obscure. Inflorescence 1-2 per axil. Flowers about $5 \mathrm{~mm}$ across, pale purple or yellow with reddish spot; pedicellate; pedicel 1.5-1.8 $\mathrm{cm}$ long, hairy along one side. Sepals: lateral sepals linear, up curved, 2.0-2.5 mm long, acuminate at apex, 1-2 nerved, purplish, densely hairy; lower sepal boatshaped, 3.5-4.2 × 1.0-1.6 mm, with 4-7 wrinkles, tip curved towards outside, densely hairy, purple, spur absent. Petals pale red with deep reddish- spot: dorsal petal ovate-orbicular, 2-2.2 × 3.5-3.8 mm, dorsally keeled, keel densely covered with unicellular hairs; lateral petals united, oblong, deeply clawed, 2-lobed, $3.2-3.6 \times 2.8-3.0 \mathrm{~mm}$, basal lobe small, distal lobe round at apex, dorsal auricle prominent. Stamens 5 , cohering above pistil; filaments free up to $2 / 3$ rd their length, connate at apex; anthers pale yellow. Ovary ellipsoidal, 2.0-2.2 mm long, glabrous; style rudimentary; stigma minute. Capsule ovoid, turgid, 11-12 × 4-5 mm, acute, beaked, glabrous 5-9-seeded. Seeds 6-9, round with prominent caruncle, 1.0-1.2 ×0.3-0.4 mm, compressed, glossy-black.

Flowering and fruiting: September to December.

Habitat \& Ecology: Impatiens megamalayana is usually found in wet rocky hill slopes and open
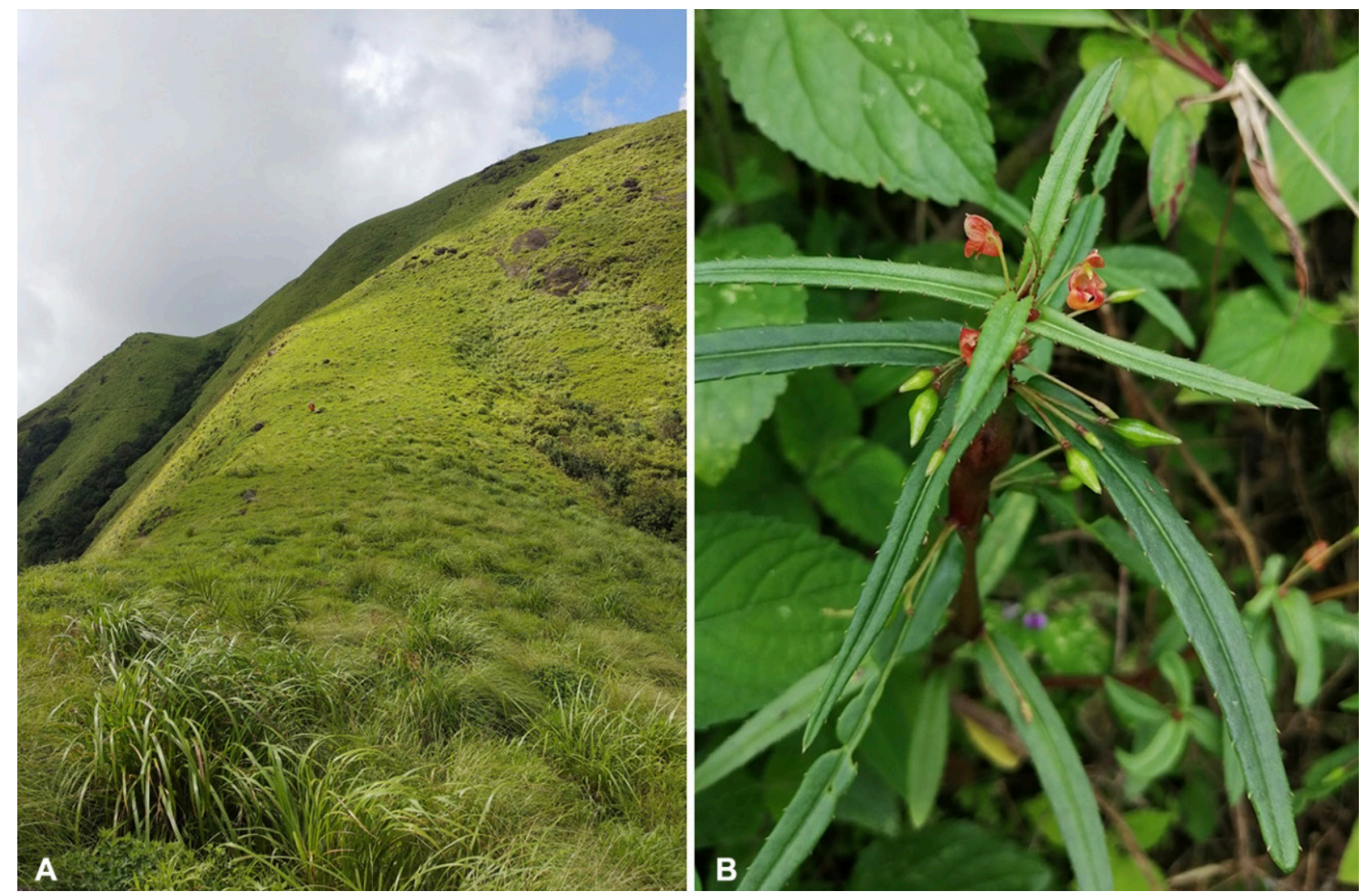

Image 1. Impatiens megamalayana: A-Habitat | B-Habit. (C) Anoop P. Balan 


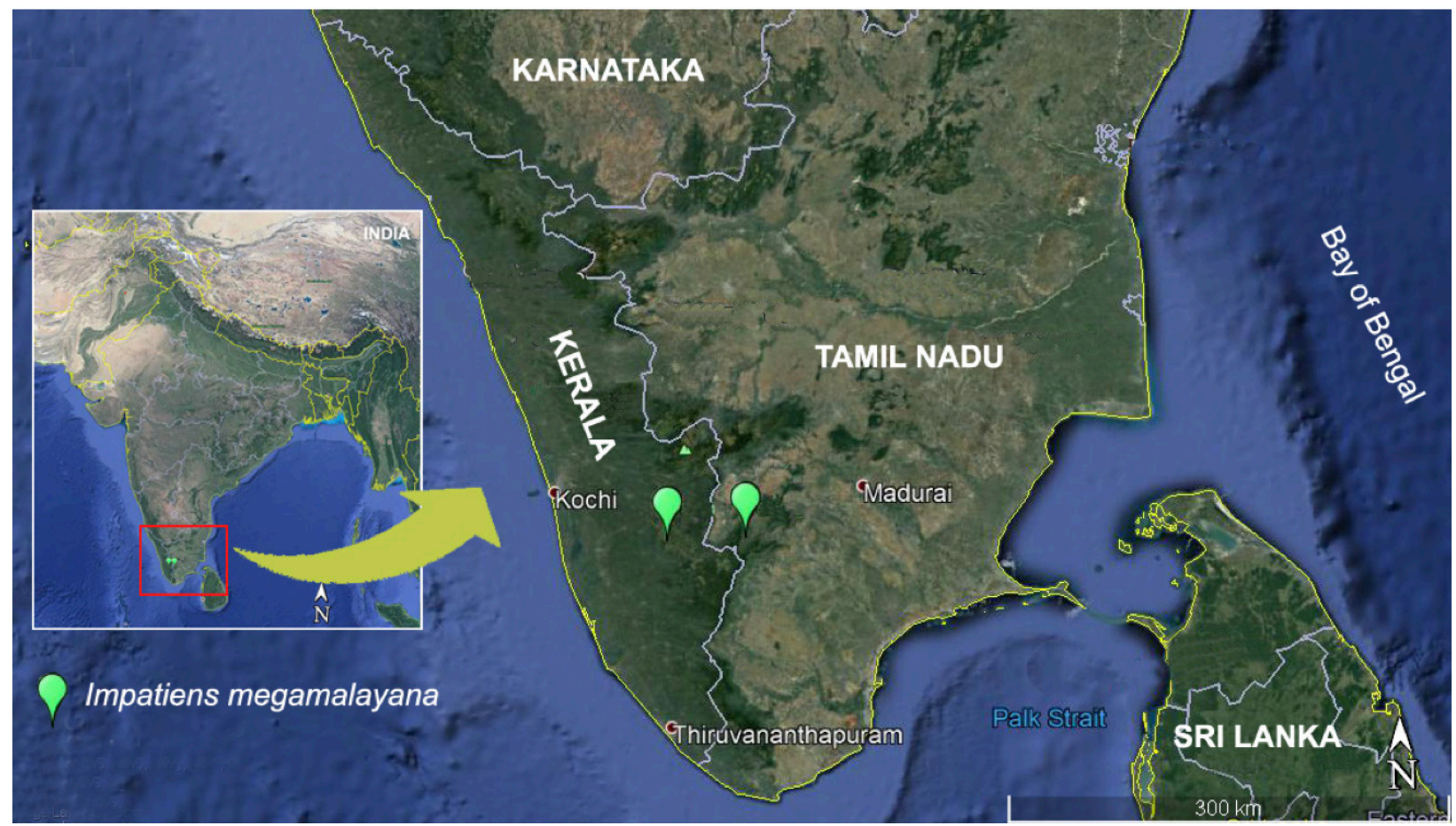

Image 2. Distribution map of Impatiens megamalayana.

grasslands at 1,000-1,200 m elevation. The plant usually grows in association with Andrographis neesiana Wight, Drosera indica L., Impatiens stolonifera Robi \& Manudev, Impatiens viscosa Bedd., Justicia procumbens L., and Smithia bigemina Dalzell.

Distribution: Endemic to the Western Ghats (Kerala \& Tamil Nadu) (Image 2).

Conservation status: Ramasubbu et al. (2017) assigned Critically Endangered status to I. megamalayana on the assumption that the taxon is strictly confined to the Megamalai hills of Tamil Nadu. However, the present report of this species from Urumbikkara hills of Kerala state necessitates a detailed field survey to assign a more appropriate IUCN status.

Notes: Urumbikkara hills and adjacent Vagamon hills are emerging tourist destinations in central Kerala. These popular hill stations are endowed with species rich evergreen forest patches, pseudo-sholas and grasslands, and are the last shelter of several Western Ghats endemic species including rare orchids and balsams. The majority of the forest patches are located outside protected forest areas, in ecologically fragile lands, and are facing severe environmental destruction due to tourism related developmental activities, illegal constructions, and encroachments. The richness of the flora of these hill stations are well evident from the recent botanical discoveries. A number of species have been described or rediscovered from these verdant hills during the last 10-12 years (Sibichen \& Nampy 2007; Robi et al. 2013; Janeesha \& Nampy 2015; Mathew et al. 2016; Manudev et al. 2017; Balan et al. 2019; Krishna et al. 2019; Balan \& Robi 2020; Balan et al. 2020). It is important to plan eco-friendly and responsible tourism projects for Urumbikkara and Vagamon to ensure the conservation of the remaining biodiversity and greenery of these beautifull hills.

Specimens examined: Kerala, Idukki District, Urumbikkara Hills, 29.viii.2020, Anoop P. Balan \& A.J. Robi 18200; 24.x.2020, Anoop P. Balan \& A.J. Robi 18202 (BAM, MBGH, MH) (Image 3).

An updated checklist of Impatiens in Kerala state and district wise distribution is provided in Table 1. Among the districts Idukki stands first with 72 out of the 107 taxa reported from Kerala of which about 22 species are strictly endemic to the district.

\section{References}

Arigela, R.K., R.K. Singh \& K.A.A. Kabeer (2019). Impatiens tanyae (Balsaminaceae), a new species from Western Ghats, India. Kew Bulletin 74(3): 48. https://doi.org/10.1007/S12225-019-9831-4

Arya S., Govind M.G., Suresh V., Vishnu W.K. \& Kumar V.N.S.A. (2021). Three new species of Impatiens (Balsaminaceae) from southern Western Ghats, Kerala. PhytoKeys 180: 157-171. https:// doi.org/10.3897/phytokeys.180.66748

Balan, A.P. \& A.J. Robi (2020). A new species and new combination in 
Table 1. An updated checklist of Impatiens in Kerala state and their district-wise distribution.

\begin{tabular}{|c|c|c|}
\hline & Name of taxa & Distribution in Kerala \\
\hline 1 & Impatiens acaulis Arn. & IDK, KNR, MPM, PKD, WND \\
\hline 2 & $\begin{array}{l}\text { I. achudanandanii V.S.A.Kumar, } \\
\text { M.G.Govind \& Sindhu Arya }\end{array}$ & TVM (Endemic) \\
\hline 3 & $\begin{array}{l}\text { I. agastyamalayensis (Bhaskar) } \\
\text { A.Joe, V.Bhaskar \& M.Sabu }\end{array}$ & TVM (Endemic) \\
\hline 4 & I. aliciae C.E.C. Fisch. & IDK (Endemic) \\
\hline 5 & I. anaimudica C.E.C. Fisch. & IDK \\
\hline 6 & I. auriculata Wight & KTM, TVM \\
\hline 7 & I. balsamina $\mathrm{L}$. & All districts \\
\hline 8 & I. brittoi B.Mani \& Sinj. Thomas & IDK (Endemic) \\
\hline 9 & I. campanulata Wight & IDK \\
\hline 10 & I. chandrasekharanii Chandrab. & IDK \\
\hline 11 & I. chinensis $\mathrm{L}$. & $\begin{array}{l}\text { IDK, KLM, KNR, PAT, PKD, TSR, } \\
\text { TVM, WND }\end{array}$ \\
\hline 12 & I. clavicornu Turcz. & IDK \\
\hline 13 & I. cochinica Hook. f. & EKM (Endemic) \\
\hline 14 & I. coelotropis C.E.C. Fisch. & IDK, PKD (Endemic) \\
\hline 15 & I. concinna Hook. f. & IDK (Endemic) \\
\hline 16 & I. cordata Wight & IDK, KKD, KLM, TVM, WND \\
\hline 17 & I. cuspidata Wight \& Arn. & IDK \\
\hline 18 & $\begin{array}{l}\text { I. danii Sindhu Arya, V.Suresh \& } \\
\text { V.S.A.Kumar }\end{array}$ & IDK (Endemic) \\
\hline 19 & I. dasysperma Wight & $\begin{array}{l}\text { IDK, KKD, KNR, MPM, PKD, } \\
\text { WND }\end{array}$ \\
\hline 20 & I. denisonii Bedd. & IDK, PKD, WND, (Endemic) \\
\hline 21 & I. disotis Hook.f. & IDK, KSD \\
\hline 22 & $\begin{array}{l}\text { I. diversifolia Wall. ex Wight \& } \\
\text { Arn. }\end{array}$ & $\begin{array}{l}\text { ALP, KLM, KNR, TSR, TVM, } \\
\text { WND }\end{array}$ \\
\hline 23 & I. elegans Bedd. & IDK \\
\hline 24 & $\begin{array}{l}\text { I. eravikulamensis Hareesh \& } \\
\text { Salish }\end{array}$ & IDK (Endemic) \\
\hline 25 & I. flaccida Arn. & $\begin{array}{l}\text { IDK, KKD, KLM, KNR, MPM, } \\
\text { PAT, PKD, TSR }\end{array}$ \\
\hline 26 & I. floribunda Wight & KLM, KSD, PKD, TVM \\
\hline 27 & I. fruticosa Lesch. ex DC. & IDK, KLM \\
\hline 28 & I. gardneriana Wight & $\begin{array}{l}\text { KKD, KNR, KTM, MPM, PKD, } \\
\text { WND }\end{array}$ \\
\hline 29 & $\begin{array}{l}\text { I. glabrata K.M.P.Kumar, Hareesh } \\
\text { \& Bhaskar }\end{array}$ & PKD (Endemic) \\
\hline 30 & I. goughii Wight & IDK, KNR, PAT, PKD, TSR \\
\hline 31 & I. grandis B.Heyne & IDK \\
\hline 32 & $\begin{array}{l}\text { 1. grandispora Nampy \& } \\
\text { M.Vishnu }\end{array}$ & IDK (Endemic) \\
\hline 33 & I. hensloviana Arn. & IDK, KNR, PKD, TSR, TVM \\
\hline 34 & I. herbicola Hook. f. & $\begin{array}{l}\text { IDK, KNR, KTM, PAT, PKD, } \\
\text { WND }\end{array}$ \\
\hline 35 & $\begin{array}{l}\text { I. inconspicua Benth. ex Wight } \\
\text { \& Arn. }\end{array}$ & $\begin{array}{l}\text { IDK, KLM, KNR, PAT, PKD, } \\
\text { TVM, WND }\end{array}$ \\
\hline 36 & I. jerdoniae Wight & IDK, KNR, PKD, WND \\
\hline 37 & I. johnii E.Barnes & IDK (Endemic) \\
\hline 38 & $\begin{array}{l}\text { I. johnsiana Ratheesh, Sunil \& } \\
\text { Anil }\end{array}$ & WND (Endemic) \\
\hline 39 & $\begin{array}{l}\text { I. josephia Sinj.Thomas, B.Mani } \\
\text { \& Britto }\end{array}$ & IDK (Endemic) \\
\hline
\end{tabular}

\begin{tabular}{|c|c|c|}
\hline 40 & $\begin{array}{l}\text { I. kulamavuensis Pandur. \& V.J. } \\
\text { Nair }\end{array}$ & IDK (Endemic) \\
\hline 41 & I. Iaticornis C.E.C. Fisch. & IDK \\
\hline 42 & I. Iatifolia L. & IDK, TVM \\
\hline 43 & I. lawsonii Hook. f. & KNR, WND \\
\hline 44 & I. leptura Hook. f. & IDK, KLM \\
\hline 45 & I. leschenaultia (DC.) Wall. & MPM, PKD \\
\hline 46 & I. levingei Gamble ex Hook. f. & IDK, TVM \\
\hline 47 & I. ligulata Bedd. & PKD, TSR \\
\hline 48 & I. macrocarpa Hook.f. & IDK (Endemic) \\
\hline 49 & I. maculata Wight & IDK, KLM, PKD \\
\hline 50 & $\begin{array}{l}\text { I. mankulamensis K.M.P.Kumar, } \\
\text { R.Jagad. \& Nagaraj }\end{array}$ & IDK (Endemic) \\
\hline 51 & I. matthewiana Ramas. \& Pandur. & IDK (Endemic) \\
\hline 52 & I. megamalayana Ramas. & IDK \\
\hline 53 & $\begin{array}{l}\text { I. minae Ratheesh, Anil Kumar } \\
\text { \& Sivad. }\end{array}$ & WND (Endemic) \\
\hline 54 & I. minor (DC.) Bennet & $\begin{array}{l}\text { All districts except ALP, EKM, } \\
\text { TVM, }\end{array}$ \\
\hline 55 & I. modesta Wight & IDK, PKD, TVM \\
\hline 56 & $\begin{array}{l}\text { I. mohana Ratheesh, Sujana \& } \\
\text { Anil Kumar }\end{array}$ & WND (Endemic) \\
\hline 57 & I. munnarensis E.Barnes & IDK (Endemic) \\
\hline 58 & I. munronii Wight & PKD \\
\hline 59 & I. neo-barnesii C.E.C. Fisch. & PKD \\
\hline 60 & $\begin{array}{l}\text { I. neo-modesta Hareesh, } \\
\text { K.M.P.Kumar \& V.B.Sreek. }\end{array}$ & PKD (Endemic) \\
\hline 61 & $\begin{array}{l}\text { I. nidholapathra M.Vishnu \& } \\
\text { Nampy }\end{array}$ & IDK (Endemic) \\
\hline 62 & I. nilgirica C.E.C. Fisch. & IDK \\
\hline 63 & 1. oppositifolia L. & IDK, PKD, TSR, WND \\
\hline 64 & I. orchioides Bedd. & IDK \\
\hline 65 & I. pallidiflora Hook.f. & IDK (Endemic) \\
\hline 66 & 1. pandata E.Barnes & IDK (Endemic) \\
\hline 67 & $\begin{array}{l}\text { I. panduranganii K.M.P.Kumar, } \\
\text { R.Jagad. \& G.Prasad }\end{array}$ & IDK (Endemic) \\
\hline 68 & I. parasitica Bedd. & IDK, KNR, PKD, WND \\
\hline 69 & I. parvifolia Bedd. & IDK, WND \\
\hline 70 & $\begin{array}{l}\text { I. pendula B.Heyne ex Wight } \\
\text { \& Arn. }\end{array}$ & IDK \\
\hline 71 & $\begin{array}{l}\text { I. periyarensis B.Mani, Sinj. } \\
\text { Thomas \& Britto }\end{array}$ & IDK (Endemic) \\
\hline 72 & I. phoenicea Bedd. & IDK \\
\hline 73 & I. platyadena C.E.C. Fisch. & IDK (Endemic) \\
\hline 74 & I. pseudoacaulis Bhaskar & PKD \\
\hline 75 & I. pulcherrima Dalz. & IDK, PKD \\
\hline 76 & I. raktakesara Vishnu \& Nampy & IDK (Endemic) \\
\hline 77 & I. repens Moon ex Wight & All districts \\
\hline 78 & $\begin{array}{l}\text { I. rufescens Benth. ex Wight \& } \\
\text { Arn. }\end{array}$ & TVM \\
\hline 79 & $\begin{array}{l}\text { I. saulierea B.Mani, Sinj.Thomas } \\
\text { \& Britto }\end{array}$ & KKD (Endemic) \\
\hline
\end{tabular}




\begin{tabular}{|c|c|c|}
\hline 80 & $\begin{array}{l}\text { I. sahyadrica V.B. Sreek., } \\
\text { Hareesh, Dantas \& Sujanapal }\end{array}$ & PKD (Endemic) \\
\hline 81 & $\begin{array}{l}\text { I. sasidharanii K.M.P. Kumar, } \\
\text { Omalsree, Hareesh \& V.B.Sreek. }\end{array}$ & PKD (Endemic) \\
\hline 82 & $\begin{array}{l}\text { I. sasidharanii var. hirsuta } \\
\text { K.M.P.Kumar, Omalsree, Hareesh } \\
\text { \& V.B.Sreek. }\end{array}$ & PKD (Endemic) \\
\hline 83 & I. scabriuscula B.Heyne ex Roxb. & KNR, PKD, WND \\
\hline 84 & I. scapiflora B.Heyne ex Roxb. & $\begin{array}{l}\text { All districts except ALP, EKM, } \\
\text { KLM, KTM }\end{array}$ \\
\hline 85 & $\begin{array}{l}\text { I. shailajae Sindhu Arya \& } \\
\text { V.S.A.Kumar }\end{array}$ & TVM (Endemic) \\
\hline 86 & $\begin{array}{l}\text { I. sholayarensis M.Kumar \& } \\
\text { Sequiera }\end{array}$ & TSR (Endemic) \\
\hline 87 & I. sivarajanii M.Kumar \& Sequiera & PKD (Endemic) \\
\hline 88 & I. stocksii Hook. f. \& Thomson & IDK, KNR, WND \\
\hline 89 & I. stolonifera Robi \& Manudev & IDK, KTM (Endemic) \\
\hline 90 & I. tangachee Bedd. & IDK \\
\hline 91 & I. tenella B.Heyne ex Hook.f. & PKD \\
\hline 92 & $\begin{array}{l}\text { I. theuerkaufiana Ratheesh \& } \\
\text { Sivad. }\end{array}$ & WND \\
\hline 93 & I. tilo (DC.) Suresh & KKD (Endemic) \\
\hline 94 & $\begin{array}{l}\text { I. tomentosa B.Heyne ex Wight } \\
\text { \& Arn. }\end{array}$ & IDK, PAT, TVM \\
\hline 95 & I. travancorica Bedd. & TVM \\
\hline 96 & I. trichocarpa Hook. f. & IDK \\
\hline 97 & I. umbellata B.Heyne & TVM \\
\hline 98 & 1. uncinata Wight & IDK, TVM \\
\hline 99 & $\begin{array}{l}\text { I. veerapazhasii Ratheesh, } \\
\text { Sujanapal \& Meera }\end{array}$ & KNR, WND (Endemic) \\
\hline 100 & I. verecunda Hook. f. & IDK (Endemic) \\
\hline 101 & I. verticillata Wight & IDK, PAT, TSR, TVM \\
\hline 102 & I. violacea M.Kumar \& Sequiera & IDK (Endemic) \\
\hline 103 & I. viridiflora Wight & IDK, PKD \\
\hline 104 & I. viscida Wight & TVM \\
\hline 105 & I. viscosa Bedd. & IDK, KTM, PAT, PKD, WND \\
\hline 106 & I. walleriana Hook.f. & All districts \\
\hline 107 & I. wightiana Bedd. & IDK,PKD, TSR (Endemic) \\
\hline
\end{tabular}
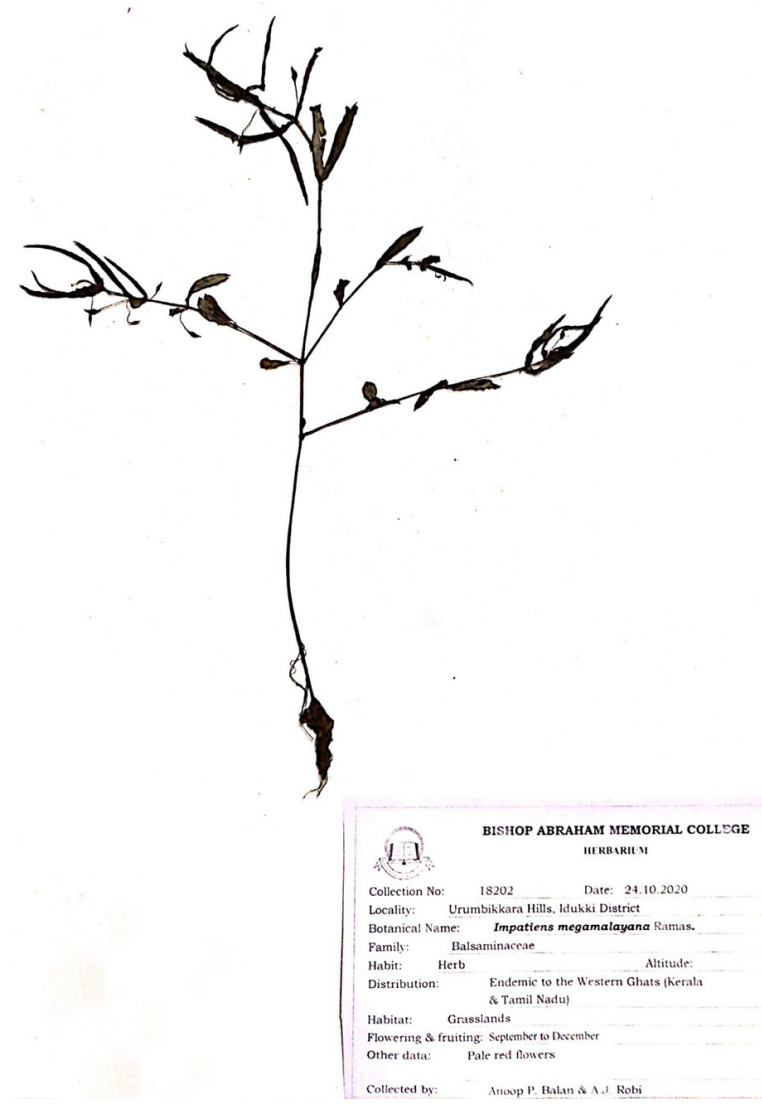

Image 3. Herbarium sheet of Impatiens megamalayana (\# 18202)

Bhaskar, V. (2012). Taxonomic monograph on Impatiens L. (Balsaminaceae) of Western Ghats, south India: the key genus for endemism. Centre for plant taxonomic studies, Bangalore, 283pp.

Chhabra, T., R. Singh, K.M. Prabhukumar \& V.S. Hareesh (2016). Three new taxa of Impatiens (Balsaminaceae) from southern Western Ghats, India. Nordic Journal of Botany 34: 708-717. https://doi. org/10.1111/njb.01139

Dessai, J.R.N. \& M.K. Janarthanam (2011). The genus Impatiens (Balsaminaceae) in the northern and parts of central Western Ghats. Rheedea 21: 23-80.

ALP-Alappuzha | EKM-Eranakulam | IDK-Idukki | KKD-Kozhikode | KLM-Kollam | KNR-Kannur | KSD-Kasargod | KTM-Kottayam | MPMMalappuram | PAT-Pathanamthitta | PKD-Palakkad | TSR-Thrissur | TVMThiruvananthapuram | WND-Wayanad.

Indian Gymnanthemum (Vernonieae, Asteraceae). Annales Botanici Fennici 57: 317-319. https://doi.org/10.5735/085.057.0428

Balan, A.P., A.J. Robi \& G. Joseph (2020). Ophiorrhiza meenachilarensis, a new species of Rubiaceae from southern Western Ghats, India. Webbia 75(2): 231-236. https://doi.org/10.36253/jopt-8508

Balan, A.P., A.J. Robi \& S.V. Predeep (2019). Notes on the extended distribution of Humboldtia bourdillonii (Fabales: Fabaceae), an Endangered tree legume in the Western Ghats, India. Journal of Threatened Taxa 11(14): 14886-14890. https://doi.org/10.11609/ jott.5424.11.14.14886-14890

Bhaskar, V. \& A.N. Sringeswara (2017). Two new species of Impatiens L. under the section: 'Annuae' (Balsaminaceae) from Western Ghats, India. Webbia 72(2): 165-170. https://doi.org/10.1080/008 37792.2017.1370208

Grey-Wilson, C. (1980). Impatiens of Africa. Morphology, pollination and pollinators, ecology phytogeography, hybridiasation, keys and a systematic treatment of all African species, with a note on collection and conservation. A.A. Balkema, Rotterdam.

Hareesh, V.S., C.V. Sanal, S. Sabik \& V.B. Sreekumar (2015b). A new distributional record of Impatiens pseudo-acaulis (Balsaminaceae) from Western Ghats of Kerala. Zoo's Print 30(9): 12-13.

Hareesh, V.S., V.B. Sreekumar, K.J. Dantas \& P. Sujanapal (2015a). Impatiens sahyadrica sp. nov. (Balsaminaceae)-a new species from southern Western Ghats, India. Phytotaxa 207(3): 291-296. https:// doi.org/10.11646/phytotaxa.207.3.8

Janeesha, M. \& S. Nampy (2015). Henckelia bracteata, a new species of Gesneriaceae from S. Western Ghats, India, and lectotypification of Didymocarpus humboldtianus (H. humboldtiana). Willdenowia 45(1): 53-59. https://doi.org/10.3372/wi.45.45105

Joe, A., V. Bhaskar \& M. Sabu (2017). Impatiens agastyamalayensis stat. nov. (Balsaminaceae)-A reassessement of Impatiens rufescens var. agastyamalayensis and rediscovery of the plant fom the Western Ghats, India. Phytotaxa 326 (20): 144-150. https://doi. org/10.11646/phytotaxa.326.2.6

Krishna, N., S.J. Britto, S. Thomas, B. Mani, A.K. Pradeep \& K.V. 
Jithin (2019). A new section (Begonia sect. Flocciferae sect. Nov.) and two new species in Begoniaceae from the Western Ghats of India. Edinburgh Journal of Botany 77(2): 251-268. https://doi. org/10.1017/S0960428619000349

Kumar, N.A., M.K.R. Narayanan, P. Sujanapal, R.M. Raj, K.A. Sujana \& Mithunlal (2011). Impatiens veerapazhasi (Balsaminaceae), a new scapigerous balasam from Wayanad, Western Ghats, India. Journal of the Botanical Research Institute of Texas 5(1): 153-158.

Mani, B. \& S. Thomas (2017). Impatiens brittoi (Balsaminaceae) sp. nov. from the southern Western Ghats, India. Nordic Journal of Botany 35(4): 440-444.

Mani, B., S. Thomas \& S.J. Britto (2018). Two new species of Impatiens (Balsaminaceae) from the Western Ghats, India. Phytotaxa 334(3): 233-240. https://doi.org/10.11646/phytotaxa.334.3.4

Mani, B., S. Thomas \& S.J. Britto (2020). A new species of Impatiens (Balsaminaceae) and rediscovery of Impatiens aliciae from the Western Ghats of India. Taiwania 65(4): 451-455. https://doi. org/10.6165/tai.2020.65.451

Manudev, K.M., A.J. Robi \& S. Nampy (2017). Impatiens stolonifera (Balsaminaceae): A new scapigerous species from the southern Western Ghats, Kerala, India. Phytotaxa 295 (1): 071-076. https:// doi.org/10.11646/phytotaxa.295.1.6

Mathew, J., R. Yohannan \& K.V. George (2016). Phyllagathis Blume (Melastomataceae: Sonerileae), a new generic record for India with a new species. Botany Letters 163(2): 175-179. https://doi.org/10.1 080/23818107.2016.1166453

Narayanan, M.K.R., J.P. Joseph, N.A. Kumar, M. Sivadasan \& A.H. Alfarhan (2013). Impatiens theuerkaufiana (Balsaminaceae), a new scapigerous species from the Western Ghats, India. Phytotaxa 83(1): 54-60. https://doi.org/10.11646/phytotaxa.83.1.3

Narayanan, M.K.R., K.A. Sujana, V. Balakrishnan, R.M. Raj \& N.A Kumar (2012b). Impatiens mohana (Balsaminaceae), a new scapigerous species from Wayanad, Eestern Ghats, India. Edinburgh Journal of Botany 69(2): 281-285. https://doi.org/10.1017/ S096042861200008X

Narayanan, M.K.R., N.A. Kumar \& J.P. Joseph (2012a). Impatiens johnsiana (Balsaminaceae), a new scapigerous species from Western Ghats, India. Journal of the Botanical Research Institute of Texas 6(1): 113-118.

Narayanan, M.K.R., N.A. Kumar, R.M. Raj, M. Sivadasan \& A.H. Alfarhan (2011). A new scapigerous species of Impatiens (Balsaminaceae) from India. Bangladesh Journal of Plant Taxonomy 18(2): 141-148.

Nayar, T.S., A.R. Beegam \& M. Sibi (2014). Flowering Plants of the Western Ghats, India, Vol. 1, pp. 195-208. Jawaharlal Nehru Tropical Botanic Garden and Research Institute, Thiruvananthapuram.

Prabhukumar, K.M., M. Omalsree, V.S. Hareesh, V. Bhaskar, T.K. Nirmesh, V.B. Sreekumar \& I. Balachandran (2015a). Two new taxa of Impatiens L. (Balsaminaceae) from Western Ghats of India. Phytotaxa 238(3): 255-264. https://doi.org/10.11646/ phytotaxa.238.3.4

Prabhukumar, K.M., R. Jagadeesan, G. Prasad, B.A.N. Nagaraj, V.V. Naveenkumar, V. Bhaskar \& I. Balachandran (2017). Two new taxa of Impatiens (Balsaminaceae) from southern parts of Western Ghats, India. Phytotaxa 296(3): 281-286. https://doi.org/10.11646/ phytotaxa.296.3.7
Prabhukumar, K.M., V.S. Hareesh, V. Bhaskar, V.B. Sreekumar, T.K. Nirmesh \& I. Balachandran (2016). Impatiens glabrata (Balsaminaceae)-A new species from southern Western Ghats, India. Phytotaxa 266: 33-39. https://doi.org/10.11646/ phytotaxa.266.1.5

Prabhukumar, K.M., V.S. Hareesh, V.B. Sreekumar, T.K. Nirmesh \& I. Balachandran (2015b). Impatiens neo-modesta (Balsaminaceae): a new species from Western Ghats, India. Webbia. 70: 231-235. https://doi.org/10.1080/00837792.2015.1074792

Ramasubbu, R., A. Surendran \& A.C. Prabha (2020a). Two new species of Impatiens (Balsaminaceae) from the Western Ghats of Tamil Nadu, India. Phytotaxa 460(4): 249-258. https://doi.org/10.11646/ phytotaxa.460.4.2

Ramasubbu, R., A. Surendran \& A.C. Prabha (2020b). A new species Impatiens L. (Balsaminaceae) from Kodaikkanal Wildlife sanctuary, India. Taiwania 65(4): 426-430. https://doi.org/10.6165/ tai.2020.65.426

Ramasubbu, R., C. Divya, N.S. Kala, A. Surendran \& A.K. Sreekala (2017). Impatiens megamalayana, a new species of Impatiens from the Western Ghats, Tamil Nadu, India. Phytotaxa 302(2): 193-197. https://doi.org/10.11646/phytotaxa.302.2.10

Ramasubbu, R., F. Irudhyaraj, D. Mohanraj, A. \& A.G. Pandurangan (2015b). Impatiens matthewiana, a new scapigerous balsam from Western Ghats, India. Phytotaxa 227(3): 268-274. https://doi. org/10.11646/phytotaxa.227.3.6

Ramasubbu, R., G. Manikandan, P. Mehalingam \& A.G. Pandurangan (2015a). Impatiens courtallensis (Balsaminaceae), a new species of Impatiens from the Western Ghats, Tamil Nadu, India. Phytotaxa 203(2): 199-204. https://doi.org/10.11646/phytotaxa.203.2.10

Robi, A.J., J. Augastine, N. Sasidharan \& P.S. Udayan (2013). Rediscovery of an endemic and rare Premna paucinervis (Verbenaceae) from the Western Ghats after a century. Rheedea 23(1): 10-12.

Salish, M.J., V.S. Hareesh, L. Arun, S. Sandeep \& P. Rajan (2019). A new species of Impatiens (Balsaminaceae) from Eravikulam National Park, southern Western Ghats, India. Webbia 74(2): 271274. https://doi.org/10.1080/00837792.2019.1637176

Sasidharan N. (2011). Flowering Plants of Kerala: CD-ROM ver. 2.0. Kerala Forest Research Institute, Peechi, Kerala.

Sibichen, M.T. \& S. Nampy (2007). Crotalaria kurisumalayana Sibichen \& Nampy (Fabaceae), a new species from India. Candollea 62: 105108.

Subbiah, K. \& R. Vellingiri (2019). Impatiens flavescens sp. nov. (Balsaminaceae), a first yellow flowered balsam from the southern Western Ghats of India. Webbia 74(2): 265-269. https://doi.org/10. 1080/00837792.2019.1659547

Vishnu, M., D. Francis, S. Nampy \& Divya K. Venugopal (2021). Impatiens raktakesara (Balsaminaceae), a new species from the southern Western Ghats, India. Brittonia 20(10): 1-7. https://doi. org/10.1007/s12228-021-09676-7

Vishnu, M., D.K. Venugopal, D. Francis \& S. Nampy (2020). Two new scapigerous species of Impatiens (Balsaminaceae) from southern Western Ghats, India. Taiwania 65(2): 187-194. https://doi. org/10.6165/tai.2020.65.187

Yuan, Y-M., Y. Song, K. Geuten, E. Rahelivololona, S. Wohlhauser, E. Fischer, E. Smets \& P. Kupfer (2004). Phylogeny and biogeography of Balsaminaceae inferred from ITS sequences. Taxon 53: 391-403. https://doi.org/10.2307/4135617 
Dr. Albert G. Orr, Griffith University, Nathan, Australia

Dr. Sameer Padhye, Katholieke Universiteit Leuven, Belgium

Dr. Nancy van der Poorten, Toronto, Canada

Dr. Kareen Schnabel, NIWA, Wellington, New Zealand

Dr. R.M. Sharma, (Retd.) Scientist, Zoological Survey of India, Pune, India

Dr. Manju Siliwal, WILD, Coimbatore, Tamil Nadu, India

Dr. G.P. Sinha, Botanical Survey of India, Allahabad, India

Dr. K.A. Subramanian, Zoological Survey of India, New Alipore, Kolkata, India

Dr. P.M. Sureshan, Zoological Survey of India, Kozhikode, Kerala, India

Dr. R. Varatharajan, Manipur University, Imphal, Manipur, India

Dr. Eduard Vives, Museu de Ciències Naturals de Barcelona, Terrassa, Spain

Dr. James Young, Hong Kong Lepidopterists' Society, Hong Kong

Dr. R. Sundararaj, Institute of Wood Science \& Technology, Bengaluru, India

Dr. M. Nithyanandan, Environmental Department, La Ala Al Kuwait Real Estate. Co. K.S.C., Kuwait

Dr. Himender Bharti, Punjabi University, Punjab, India

Mr. Purnendu Roy, London, UK

Dr. Saito Motoki, The Butterfly Society of Japan, Tokyo, Japan

Dr. Sanjay Sondhi, TITLI TRUST, Kalpavriksh, Dehradun, India

Dr. Nguyen Thi Phuong Lien, Vietnam Academy of Science and Technology, Hanoi, Vietnam

Dr. Nitin Kulkarni, Tropical Research Institute, Jabalpur, India

Dr. Robin Wen Jiang Ngiam, National Parks Board, Singapore

Dr. Lional Monod, Natural History Museum of Geneva, Genève, Switzerland.

Dr. Asheesh Shivam, Nehru Gram Bharti University, Allahabad, India

Dr. Rosana Moreira da Rocha, Universidade Federal do Paraná, Curitiba, Brasil

Dr. Kurt R. Arnold, North Dakota State University, Saxony, Germany

Dr. James M. Carpenter, American Museum of Natural History, New York, USA

Dr. David M. Claborn, Missouri State University, Springfield, USA

Dr. Kareen Schnabel, Marine Biologist, Wellington, New Zealand

Dr. Amazonas Chagas Júnior, Universidade Federal de Mato Grosso, Cuiabá, Brasil

Mr. Monsoon Jyoti Gogoi, Assam University, Silchar, Assam, India

Dr. Heo Chong Chin, Universiti Teknologi MARA (UiTM), Selangor, Malaysia

Dr. R.J. Shiel, University of Adelaide, SA 5005, Australia

Dr. Siddharth Kulkarni, The George Washington University, Washington, USA

Dr. Priyadarsanan Dharma Rajan, ATREE, Bengaluru, India

Dr. Phil Alderslade, CSIRO Marine And Atmospheric Research, Hobart, Australia

Dr. John E.N. Veron, Coral Reef Research, Townsville, Australia

Dr. Daniel Whitmore, State Museum of Natural History Stuttgart, Rosenstein, Germany.

Dr. Yu-Feng Hsu, National Taiwan Normal University, Taipei City, Taiwan

Dr. Keith V. Wolfe, Antioch, California, USA

Dr. Siddharth Kulkarni, The Hormiga Lab, The George Washington University, Washington,

D.C., USA

Dr. Tomas Ditrich, Faculty of Education, University of South Bohemia in Ceske

Budejovice, Czech Republic

Dr. Mihaly Foldvari, Natural History Museum, University of Oslo, Norway

Dr. V.P. Uniyal, Wildlife Institute of India, Dehradun, Uttarakhand 248001, India

Dr. John T.D. Caleb, Zoological Survey of India, Kolkata, West Bengal, India

Dr. Priyadarsanan Dharma Rajan, Ashoka Trust for Research in Ecology and the Environment (ATREE), Royal Enclave, Bangalore, Karnataka, India

\section{Fishes}

Dr. Neelesh Dahanukar, IISER, Pune, Maharashtra, India

Dr. Topiltzin Contreras MacBeath, Universidad Autónoma del estado de Morelos, México

Dr. Heok Hee Ng, National University of Singapore, Science Drive, Singapore

Dr. Rajeev Raghavan, St. Albert's College, Kochi, Kerala, India

Dr. Robert D. Sluka, Chiltern Gateway Project, A Rocha UK, Southall, Middlesex, UK

Dr. E. Vivekanandan, Central Marine Fisheries Research Institute, Chennai, India

Dr. Davor Zanella, University of Zagreb, Zagreb, Croatia

Dr. A. Biju Kumar, University of Kerala, Thiruvananthapuram, Kerala, India

Dr. Akhilesh K.V., ICAR-Central Marine Fisheries Research Institute, Mumbai Research

Centre, Mumbai, Maharashtra, India

Dr. J.A. Johnson, Wildlife Institute of India, Dehradun, Uttarakhand, India

\section{Amphibians}

Dr. Sushil K. Dutta, Indian Institute of Science, Bengaluru, Karnataka, India

Dr. Annemarie Ohler, Muséum national d'Histoire naturelle, Paris, France

\section{Reptiles}

Dr. Gernot Vogel, Heidelberg, Germany

Dr. Raju Vyas, Vadodara, Gujarat, India

Dr. Pritpal S. Soorae, Environment Agency, Abu Dubai, UAE.

Prof. Dr. Wayne J. Fuller, Near East University, Mersin, Turkey

Prof. Chandrashekher U. Rivonker, Goa University, Taleigao Plateau, Goa. India

Dr. S.R. Ganesh, Chennai Snake Park, Chennai, Tamil Nadu, India

Dr. Himansu Sekhar Das, Terrestrial \& Marine Biodiversity, Abu Dhabi, UAE
Birds

Dr. Hem Sagar Baral, Charles Sturt University, NSW Australia

Dr. Chris Bowden, Royal Society for the Protection of Birds, Sandy, UK

Dr. Priya Davidar, Pondicherry University, Kalapet, Puducherry, India

Dr. J.W. Duckworth, IUCN SSC, Bath, UK

Dr. Rajah Jayapal, SACON, Coimbatore, Tamil Nadu, India

Dr. Rajiv S. Kalsi, M.L.N. College, Yamuna Nagar, Haryana, India

Dr. V. Santharam, Rishi Valley Education Centre, Chittoor Dt., Andhra Pradesh, India

Dr. S. Balachandran, Bombay Natural History Society, Mumbai, India

Mr. J. Praveen, Bengaluru, India

Dr. C. Srinivasulu, Osmania University, Hyderabad, India

Dr. K.S. Gopi Sundar, International Crane Foundation, Baraboo, USA

Dr. Gombobaatar Sundev, Professor of Ornithology, Ulaanbaatar, Mongolia

Prof. Reuven Yosef, International Birding \& Research Centre, Eilat, Israel

Dr. Taej Mundkur, Wetlands International, Wageningen, The Netherlands

Dr. Carol Inskipp, Bishop Auckland Co., Durham, UK

Dr. Tim Inskipp, Bishop Auckland Co, Durham, UK

Dr. V. Gokula, National College, Tiruchirappalli, Tamil Nadu, India

Dr. Arkady Lelej, Russian Academy of Sciences, Vladivostok, Russia

Dr. Simon Dowell, Science Director, Chester Zoo, UK

Dr. Mário Gabriel Santiago dos Santos, Universidade de Trás-os-Montes e Alto Douro,

Quinta de Prados, Vila Real, Portugal

Dr. Grant Connette, Smithsonian Institution, Royal, VA, USA

Dr. M. Zafar-ul Islam, Prince Saud Al Faisal Wildlife Research Center, Taif, Saudi Arabia

Mammals

Dr. Giovanni Amori, CNR - Institute of Ecosystem Studies, Rome, Italy

Dr. Anwaruddin Chowdhury, Guwahati, India

Dr. David Mallon, Zoological Society of London, UK

Dr. Shomita Mukherjee, SACON, Coimbatore, Tamil Nadu, India

Dr. Angie Appel, Wild Cat Network, Germany

Dr. P.O. Nameer, Kerala Agricultural University, Thrissur, Kerala, India

Dr. Ian Redmond, UNEP Convention on Migratory Species, Lansdown, UK

Dr. Heidi S. Riddle, Riddle's Elephant and Wildlife Sanctuary, Arkansas, USA

Dr. Karin Schwartz, George Mason University, Fairfax, Virginia.

Dr. Lala A.K. Singh, Bhubaneswar, Orissa, India

Dr. Mewa Singh, Mysore University, Mysore, India

Dr. Paul Racey, University of Exeter, Devon, UK

Dr. Honnavalli N. Kumara, SACON, Anaikatty P.O., Coimbatore, Tamil Nadu, India

Dr. Nishith Dharaiya, HNG University, Patan, Gujarat, India

Dr. Spartaco Gippoliti, Socio Onorario Società Italiana per la Storia della Fauna "Giuseppe

Altobello", Rome, Italy

Dr. Justus Joshua, Green Future Foundation, Tiruchirapalli, Tamil Nadu, India

Dr. H. Raghuram, The American College, Madurai, Tamil Nadu, India

Dr. Paul Bates, Harison Institute, Kent, UK

Dr. Jim Sanderson, Small Wild Cat Conservation Foundation, Hartford, USA

Dr. Dan Challender, University of Kent, Canterbury, UK

Dr. David Mallon, Manchester Metropolitan University, Derbyshire, UK

Dr. Brian L. Cypher, California State University-Stanislaus, Bakersfield, CA

Dr. S.S. Talmale, Zoological Survey of India, Pune, Maharashtra, India

Prof. Karan Bahadur Shah, Budhanilakantha Municipality, Kathmandu, Nepal

Dr. Susan Cheyne, Borneo Nature Foundation International, Palangkaraja, Indonesia

Dr. Hemanta Kafley, Wildlife Sciences, Tarleton State University, Texas, USA

\section{Other Disciplines}

Dr. Aniruddha Belsare, Columbia MO 65203, USA (Veterinary)

Dr. Mandar S. Paingankar, University of Pune, Pune, Maharashtra, India (Molecular)

Dr. Jack Tordoff, Critical Ecosystem Partnership Fund, Arlington, USA (Communities)

Dr. Ulrike Streicher, University of Oregon, Eugene, USA (Veterinary)

Dr. Hari Balasubramanian, EcoAdvisors, Nova Scotia, Canada (Communities)

Dr. Rayanna Hellem Santos Bezerra, Universidade Federal de Sergipe, São Cristóvão, Brazil

Dr. Jamie R. Wood, Landcare Research, Canterbury, New Zealand

Dr. Wendy Collinson-Jonker, Endangered Wildlife Trust, Gauteng, South Africa

Dr. Rajeshkumar G. Jani, Anand Agricultural University, Anand, Gujarat, India

Dr. O.N. Tiwari, Senior Scientist, ICAR-Indian Agricultural Research Institute (IARI), New

Delhi, India

Dr. L.D. Singla, Guru Angad Dev Veterinary and Animal Sciences University, Ludhiana, India

Dr. Rupika S. Rajakaruna, University of Peradeniya, Peradeniya, Sri Lanka

Dr. Bahar Baviskar, Wild-CER, Nagpur, Maharashtra 440013, India

Reviewers 2018-2020

Due to pausity of space, the list of reviewers for 2018-2020 is available online.
The opinions expressed by the authors do not reflect the views of the Journal of Threatened Taxa, Wildlife Information Liaison Development Society, Zoo Outreach Organization, or any of the partners. The journal, the publisher, the host, and the partners are not responsible for the accuracy of the political boundaries shown in the maps by the authors.

\footnotetext{
Print copies of the Journal are available at cost. Write to:

The Managing Editor, JoTT,

c/o Wildlife Information Liaison Development Society,

No. 12, Thiruvannamalai Nagar, Saravanampatti - Kalapatti Road,

Saravanampatti, Coimbatore, Tamil Nadu 641035, India

ravi@threatenedtaxa.org
} 


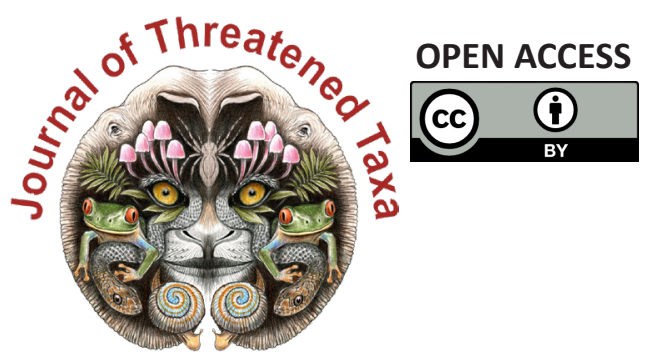

www.threatenedtaxa.org

The Journal of Threatened Taxa (JoTT) is dedicated to building evidence for conservation globally by publishing peer-reviewed articles online every month at a reasonably rapid rate at www.threatenedtaxa.org. All articles published in JoTT are registered under Creative Commons Attribution 4.0 International License unless otherwise mentioned. JoTT allows allows unrestricted use, reproduction, and distribution of articles in any medium by providing adequate credit to the author(s) and the source of publication.

\section{ISSN $0974-7907$ (Online) | ISSN $0974-7893$ (Print)}

\section{October 2021 | Vol. 13 | No. 12 | Pages: 19675-19886 \\ Date of Publication: 26 October 2021 (Online \& Print) DOI: 10.11609/jott.2021.13.12.19675-19886}

Articles

Roosting habits and habitats of the Indian Flying Fox Pteropus medius Temminck, 1825 in the northern districts of Tamil Nadu, India

- M. Pandian \& S. Suresh, Pp. 19675-19688

Diversity and distribution of avifauna at Warathenna-Hakkinda Environmental Protection Area in Kandy, Sri Lanka

- Dinelka Thilakarathne, Tithira Lakkana, Gayan Hirimuthugoda, Chaminda Wijesundara \& Shalika Kumburegama, Pp. 19689-19701

Grass species composition in tropical forest of southern India

- M. Ashokkumar, S. Swaminathan \& R. Nagarajan, Pp. 19702-19713

\section{Communications}

Habitat use and conservation threats to Wild Water Buffalo Bubalus arnee (Mammalia: Artiodactyla: Bovidae) in Koshi Tappu Wildlife Reserve, Nepal

- Reeta Khulal, Bijaya Neupane, Bijaya Dhami, Siddhartha Regmi, Ganesh Prasad Tiwari \& Manita Parajuli, Pp. 19714-19724

Get my head around owls: people perception and knowledge about owls of Andaman Islands

- Shanmugavel Sureshmarimuthu, Santhanakrishnan Babu, Nagaraj Rajeshkumar \& Honnavalli Nagaraj Kumara, Pp. 19725-19732

Abundance and diversity of threatened birds in Nangal Wetland, Punjab, India - Rajwinder Kaur \& Onkar Singh Brraich, Pp. 19733-19742

Evaluation of fish diversity and abundance in the Kabul River with comparisons between reaches above and below Kabul City, Afghanistan

- Ugyen Kelzang, Ahmad Farid Habibi \& Ryan J. Thoni, Pp. 19743-19752

New record of Myrmarachne melanocephala MacLeay, 1839 (Araneae: Salticidae) from Jharkhand, India and biogeographical implications of the co-occurrence of its ant model Tetraponera rufonigra Jerdon, 1851

- Rahul Kumar, Mirtunjay Sharma \& Ajay Kumar Sharma, Pp. 19753-19761

Diversity of spiders (Arachnida: Araneae) and the impact of pruning in Indian sandalwood plantations from Karnataka, India

-S. Padma 1 \& R. Sundararaj, Pp. 19762-19772

New records of cheilostome Bryozoa from the eastern coast of India encrusting on the exoskeleton of live horseshoe crabs of Indian Sundarbans

- Swati Das, Maria Susan Sanjay, Basudev Tripathy, C. Venkatraman \& K.A. Subramanian, Pp. 19773-19780

On the pteridophytes of Bherjan-Borajan-Padumoni Wildlife Sanctuary, Assam, India - Pranjal Borah \& Jayanta Barukial, Pp. 19781-19790

Population status of Heritiera fomes Buch.-Ham., a threatened species from Mahanadi Mangrove Wetland, India

- Sudam Charan Sahu, Manas Ranjan Mohanta \& N.H. Ravindranath, Pp. 19791-19798

Additions to the lichenized and lichenicolous fungi of Jammu \& Kashmir from Kishtwar High Altitude National Park

- Vishal Kumar, Yash Pal Sharma, Siljo Joseph, Roshinikumar Ngangom \& Sanjeeva Nayaka, Pp. 19799-19807

\section{Short Communications}

Is release of rehabilitated wildlife with embedded lead ammunition advisable? Plumbism in a Jaguar Panthera Onca (Mammalia: Carnivora: Felidae), survivor of gunshot wounds - Eduardo A. Díaz, Carolina Sáenz, E. Santiago Jiménez, David A. Egas \& Kelly Swing, Pp. 19808-19812

New record of the Sewing Needle Zipper Loach Paracanthocobitis linypha Singer \& Page, 2015 (Teleostei: Cypriniformes: Nemacheilidae) from the Chindwin drainage of Manipur, India

- Yumnam Rameshori, Yengkhom Chinglemba \& Waikhom Vishwanath, Pp. 19813-19817

Field identification characters to diagnose Microhyla mukhlesuri from closely related M. mymensinghensis (Amphibia: Microhylidae) and range extension of $M$. mukhlesuri up to West Bengal State, India

- Suman Pratihar \& Kaushik Deuti, Pp. 19818-19823
First report of Scipinia horrida (Stål) (Heteroptera: Reduviidae) from Assam, with comments on related genus Irantha Stål

- Anjana Singha Naorem, Santana Saikia, Anandita Buragohain, Rubina Azmeera Begum, Swapnil S. Boyane \& Hemant V. Ghate, Pp. 19824-19830

Flesh fly (Diptera: Sarcophagidae): male terminalia, diversity and expanded geographical distribution from India

- Kanholi Sreejith, Shuvra Kanti Sinha, Santanu Mahato \& Edamana Pushpalatha, Pp. 1983119836

Checklist of moths (Heterocera) of Tadong, Sikkim, India

- Prayash Chettri, Yuki Matsui, Hideshi Naka \& Archana Tiwari, Pp. 19837-19848

New distribution records of Begonia L., B. murina Craib and B. poilanei Kiew (Begoniaceae: Cucurbitales) for Laos

- Phongphayboun Phonepaseuth, Phetlasy Souladeth, Soulivanh Lanorsavanh, Shuichiro Tagane, Thyraphon Vongthavone \& Keooudone Souvannakhoummane Pp. 19849-19854

Notes

A recent sighting of the Stripe-backed Weasel Mustela strigidorsa (Mammalia: Carnivora: Mustelidae) in Hkakabo Razi Landscape, Myanmar

- Sai Sein Lin Oo, Tun Tun, Kyaw Myo Naing \& Paul Jeremy James Bates, Pp. 19855-19859

Are the uplifted reef beds in North Andaman letting nesting Olive Ridley Sea Turtle Lepidochelys olivacea stranded?

- Nehru Prabakaran, Anoop Raj Singh \& Vedagiri Thirumurugan, Pp. 19860-19863

First record of the orb-weaving spider Araneus tubabdominus Zhu \& Zhang, 1993 (Araneae: Araneidae) from India

- Souvik Sen, John T.D. Caleb \& Shelley Acharya, Pp. 19864-19866

The genus Catapiestus Perty, 1831 (Coleoptera: Tenebrionidae: Cnodalonini) from Arunachal Pradesh with one new record to India

- V.D. Hegde \& Sarita Yadav, Pp. 19867-19869

Rediscovery and extended distribution of Indigofera santapaui Sanjappa (Leguminosae: Papilionoideae) from the states of Maharashtra and Gujarat, India

- Kumar Vinod Chhotupuri Gosavi, Sanjay Gajanan Auti, Sharad Suresh Kambale \& Munivenkatappa Sanjappa, Pp. 19870-19873

Additional distribution records of Ceropegia anjanerica, an endemic and 'Endangered' lantern flower of the northern Western Ghats, India

- Samir Shrikant Maity, Ajay Natha Gangurde, Sharad Suresh Kambale, Avinash Ramchandra Gholave, Avinash Asraji Adsul, Ganesh Babaso Pawar \& Kumar Vinod Chhotupuri Gosavi, Pp. 19874-19877

Notes on the extended distribution of Impatiens megamalayana, a recently described balsam in Western Ghats, India

- Anoop P. Balan \& A.J. Robi, Pp. 19878-19883

Book Review

A look over on the scented tree of India (Santalum album - S. Suresh Ramanan \& A. Arunachalam, Pp. 19884-19886
Publisher \& Host
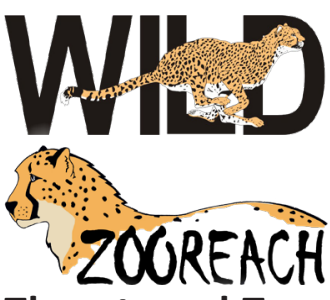

Threatened Taxa 